\title{
$\varphi$-Multipliers on Banach Algebras and Topological Modules
}

\author{
Marjan Adib \\ Department of Mathematics, Payame Noor University (PNU), P.O. Box 19395-3697, Tehran, Iran \\ Correspondence should be addressed to Marjan Adib; marjan_ad_1360@yahoo.com
}

Received 26 October 2014; Accepted 29 December 2014

Academic Editor: Antonio M. Peralta

Copyright (C) 2015 Marjan Adib. This is an open access article distributed under the Creative Commons Attribution License, which permits unrestricted use, distribution, and reproduction in any medium, provided the original work is properly cited.

We prove some results concerning Arens regularity and amenability of the Banach algebra $M_{\varphi}(A)$ of all $\varphi$-multipliers on a given Banach algebra $A$. We also consider $\varphi$-multipliers in the general topological module setting and investigate some of their properties. We discuss the $\varphi$-strict and $\varphi$-uniform topologies on $M_{\varphi}(A)$. A characterization of $\varphi$-multipliers on $L_{1}(G)$-module $L_{p}(G)$, where $G$ is a compact group, is given.

\section{Introduction}

The concept of a multiplier was introduced by Helgason [1] as follows. Let $A$ be a commutative and semisimple Banach algebra and let $\Delta(A)$ be its maximal ideal space. Let $\widehat{A}$ denote the Gelfand representation of $A$ as a subalgebra of the algebra of continuous functions on $\Delta(A)$. A bounded continuous function $g$ on $\Delta(A)$ is a multiplier on $A$ if $g \widehat{A} \subseteq \widehat{A}$. The general theory of multipliers on faithful Banach algebras was developed by Wang [2] and Birtel [3].

Recall that a mapping $T: A \rightarrow A$ is called a left (resp., right) multiplier on $A$ if

$$
T(x y)=T(x) y \quad \text { (resp., } T(x y)=x T(y))
$$

for all $x, y \in A$. We say $T$ is a multiplier on $A$ if it is both a left multiplier and a right multiplier on $A$.

We denote with $M(A)$ the algebra of all multipliers on $A$.

A Banach algebra $A$ is called left (resp., right) faithful if, for all $x \in A, x A=\{0\}$ (resp., $A x=\{0\}$ ) implies that $x=0 ; A$ is called faithful if it is both left and right faithful.

In [4] we generalized the concept of multipliers on faithful Banach algebras to $\varphi$-multipliers as follows. Let $A$ be a Banach algebra and let $\varphi: A \rightarrow A$ be an algebra homomorphism. A linear continuous mapping $T: A \rightarrow A$ is called a left (resp., right) $\varphi$-multiplier on $A$ if

$$
T(x y)=T(x) \varphi(y) \quad(\text { resp., } T(x y)=\varphi(x) T(y))
$$

for all $x, y \in A$. We say $T$ is a $\varphi$-multiplier on $A$ if it is both a left $\varphi$-multiplier and a right $\varphi$-multiplier on $A$. We denote by $M_{\varphi}(A)$ (resp., $\left.M_{\varphi}^{l}(A), M_{\varphi}^{r}(A)\right)$ the collection of all $\varphi$-multipliers (resp., left $\varphi$-multipliers, right $\varphi$-multipliers) on $A$.

It turns out that this concept is considerably more general than the concept of multipliers on Banach algebras. Also by using some well-known homomorphisms like Jordan homomorphism, spectrum preserving homomorphism, and idempotent preserving homomorphism, we can transfer these useful properties from homomorphism $\varphi$ to the algebra of $\varphi$ multipliers.

In [4], we studied various properties of $\varphi$-multipliers, for instance, the faithfulness of the Banach algebra $M_{\varphi}(A)$ and the existence of a bounded approximate identity in the range of a $\varphi$-multiplier. Finally, as an example, we have characterized $\varphi$-multipliers on $L_{1}(G)$.

In Section 2 we are concerned by Arens regularity and amenability of the Banach algebra $M_{\varphi}(A)$ under some suitable conditions. We introduce the notion of Jordan $\varphi$-multiplier and prove that every Jordan $\varphi$-multiplier is a $\varphi$-multiplier whenever the range of $\varphi$ is dense in the algebra.

In Section 3 we extend the notion of $\varphi$-multipliers on Banach algebras to topological modules and investigate some of their properties. We discuss the $\varphi$-strict and $\varphi$-uniform topologies on $M_{\varphi}(A)$ and apply our results to $L_{1}(G)$-module $L_{p}(G)$.

Let $X$ be a topological vector space and let $A$ be a topological algebra, both over the same field $\mathbb{K}(=\mathbb{R}$ or $\mathbb{C})$. Then $X$ is called a topological left $A$-module if it is a left $A$-module and the module multiplication $(a, x) \rightarrow a \cdot x$ from $A \times X$ into $X$ is separately continuous. If $b(A)$ denotes the collection of all 
bounded sets in $A$, then module multiplication $(a, x) \rightarrow a \cdot x$ is called $b(A)$-hypocontinuous [5] if, given any neighborhood $G$ of 0 in $X$ and any $D \in b(A)$, there exists a neighborhood $H$ of 0 in $X$ such that $D \cdot H \subseteq G$. Clearly, joint continuity $\Rightarrow$ hypocontinuity $\Rightarrow$ separate continuity. A mapping $\psi$ from a left $A$-module $X$ into another left $A$-module $Y$ is called an $A$ module homomorphism if $\psi(a \cdot x)=a \cdot \psi(x)$ for all $a \in A$ and $x \in X$.

\section{Some Properties of $\varphi$-Multipliers on Banach Algebras}

Let us start with the following result proved in [4].

Theorem 1 (see [4, Theorem 2.2]). Let A be a faithful commutative Banach algebra and let $\varphi$ be an idempotent homomorphism on $A$. Then $M_{\varphi}(A)$ is a Banach algebra. Moreover, if $A^{2}=A$ and $\varphi \circ T=T \circ \varphi$ for all $T \in M_{\varphi}(A)$, then $M_{\varphi}(A)$ is a faithful commutative Banach algebra.

Definition 2. Let $A$ be a Banach algebra and let $\varphi$ be a homomorphism from $A$ to $A$. The mapping $T: A \rightarrow A$ is called a left (resp., right) Jordan $\varphi$-multiplier on $A$ if for all $x \in A$

$$
T\left(x^{2}\right)=T(x) \varphi(x) \quad\left(\text { resp., } T\left(x^{2}\right)=\varphi(x) T(x)\right) .
$$

$T$ is called a Jordan $\varphi$-multiplier on $A$ if it is both a left Jordan $\varphi$-multiplier and a right Jordan $\varphi$-multiplier on $A$.

Theorem 3. Let $A$ be a faithful commutative Banach algebra and let $\varphi$ be a homomorphism from $A$ to $A$ with dense range. Then $T$ is a $\varphi$-multiplier if and only if $T$ is a Jordan $\varphi$-multiplier.

Proof. It is clear that every $\varphi$-multiplier is a Jordan $\varphi$-multiplier. Conversely, suppose $T$ is a Jordan $\varphi$-multiplier. Then

$$
\begin{aligned}
T\left((x+y)^{2}\right)= & \varphi(x+y) T(x+y) \\
= & \varphi(x) T(x)+\varphi(x) T(y) \\
& +\varphi(y) T(x)+\varphi(y) T(y)
\end{aligned}
$$

for all $x, y \in A$.

On the other hand, we have

$$
\begin{aligned}
T\left((x+y)^{2}\right) & =T\left(x^{2}+2 x y+y^{2}\right) \\
& =\varphi(x) T(x)+2 T(x y)+\varphi(y) T(y) .
\end{aligned}
$$

Comparing (4), (5) we obtain

$$
2 T(x y)=\varphi(x) T(y)+\varphi(y) T(x) .
$$

From (6) and using commutativity of $A$, for each sequence $\left\{z_{n}\right\}_{n=1}^{\infty} \subset A$ we have

$$
\begin{aligned}
2 T\left(x y z_{n}\right)= & \varphi(y) T\left(x z_{n}\right)+\varphi\left(x z_{n}\right) T(y) \\
= & \frac{\varphi(y)\left[\varphi(x) T\left(z_{n}\right)+\varphi\left(z_{n}\right) T(x)\right]}{2} \\
& +\varphi\left(x z_{n}\right) T(y),
\end{aligned}
$$

so we have

$$
\begin{aligned}
2 T\left(x y z_{n}\right)= & {\left[\varphi(y) \varphi(x) T\left(z_{n}\right)+\varphi(y) \varphi\left(z_{n}\right) T(x)\right.} \\
& \left.+2 \varphi(x) \varphi\left(z_{n}\right) T(y)\right] \cdot 2^{-1}
\end{aligned}
$$

similarly by using (6) we have

$$
\begin{gathered}
2 T\left(x y z_{n}\right)=\left[\varphi(x) \varphi(y) T\left(z_{n}\right)+\varphi(x) \varphi\left(z_{n}\right) T(y)\right. \\
\left.+2 \varphi(y) \varphi\left(z_{n}\right) T(x)\right] \cdot 2^{-1} ;
\end{gathered}
$$

comparing (8), (9) we obtain

$$
\lim _{n \rightarrow \infty} \varphi(x) \varphi\left(z_{n}\right) T(y)=\lim _{n \rightarrow \infty} \varphi(y) \varphi\left(z_{n}\right) T(x)
$$

for all $x, y, z_{n} \in A$. Since $\varphi$ has dense range and $A$ is a faithful commutative Banach algebra, we have

$$
\varphi(x) T(y)=T(x) \varphi(y)
$$

hence $T$ is a $\varphi$-multiplier.

We mention that Theorem 3 holds for certain noncommutative cases, but not in general. For instance, Zalar has proved in [6] that any left (right) Jordan multiplier on a 2torsion free semiprime ring is a left (right) multiplier. Vukman [7] has shown that an additive map $\varphi: R \rightarrow R$, where $R$ is a 2-torsion free semiprime ring, with the property that $2 \varphi\left(a^{2}\right)=a \varphi(a)+\varphi(a) a$ for all $a \in A$, is a multiplier.

The following example shows that, in general, the above theorem need not hold for noncommutative Banach algebras.

Example 4. Consider the subalgebra

$$
\mathscr{A}=\left\{\left(\begin{array}{lll}
a & b & c \\
0 & a & d \\
0 & 0 & a
\end{array}\right) \mid a, b, c, d \in \mathbb{C}\right\}
$$

of the algebra of all $3 \times 3$ matrices. It is obvious that $\mathscr{A}$ is a Banach algebra with respect to the norm given by

$$
\left\|\left(\begin{array}{lll}
a & b & c \\
0 & a & d \\
0 & 0 & a
\end{array}\right)\right\|=|a|+|b|+|c|+|d| .
$$

Let

$$
X=\left(\begin{array}{lll}
0 & 1 & 0 \\
0 & 0 & 0 \\
0 & 0 & 0
\end{array}\right)
$$

and define a continuous linear map $\varphi: \mathscr{A} \rightarrow \mathscr{A}$ by $\varphi(A)=$ $A X+X A$. By a straightforward calculation one can prove that

$$
B A X+X A B=B X A+A X B, \quad A, B \in \mathscr{A} .
$$

If ' $\circ$ ' denotes the Jordan product $A \circ B=A B+B A$, then we have $\varphi(A \circ B)=A \circ \varphi(B)$ for each $A, B \in \mathscr{A}$ and hence $\varphi$ is a 
right Jordan multiplier. Also $A \circ \varphi(B)=\varphi(1)$ for all $A, B \in \mathscr{A}$ with $A \circ B=1$. If we consider

$$
A=\left(\begin{array}{lll}
0 & 0 & 0 \\
0 & 0 & 1 \\
0 & 0 & 0
\end{array}\right)
$$

then $\varphi(A) \neq 0$ and $A \varphi(1)=0$, where 0,1 are the zero matrix and the identity matrix, respectively. Thus $\varphi$ is not a right multiplier.

Lemma 5 (see [8]). Let $A$ be an amenable Banach algebra and let $\psi$ be a continuous homomorphism of $A$ onto a dense subalgebra of a Banach algebra $B$. Then $B$ is amenable.

Theorem 6. (a) Let $A$ be a unital commutative Banach algebra and let $\varphi$ be an idempotent homomorphism on $A$ such that $\varphi$ commutes with each $S \in M_{\varphi}(A)$. If $A$ is Arens regular then $M_{\varphi}(A)$ is Arens regular.

(b) Let $A$ be a commutative Banach algebra and let $\varphi$ be as in part (a). If $A$ is amenable then $M_{\varphi}(A)$ is amenable.

Proof. (a) Define $\mu: A \rightarrow M_{\varphi}(A)$ by $\mu(a)={ }_{a} \varphi$, where ${ }_{a} \varphi(b)=\varphi(a b)$. The homomorphism $\mu$ is onto. Namely, if $S \in$ $M_{\varphi}(A)$, then $S={ }_{S(e)} \varphi$. Of course, $\mu^{* *}: A^{* *} \rightarrow\left(M_{\varphi}(A)\right)^{* *}$ has the same property, as well. Let $F^{\prime}, G^{\prime} \in\left(M_{\varphi}(A)\right)^{* *}$. Then there exist $F, G \in A^{* *}$ such that $\mu^{* *}(F)=F^{\prime}, \mu^{* *}(G)=G^{\prime}$. Let $\circ$ and $\circ^{\prime}$ be two Arens multiplications on the second dual $A^{* *}$. Thus,

$$
\begin{aligned}
F^{\prime} \circ G^{\prime} & =\mu^{* *}(F) \circ \mu^{* *}(G)=\mu^{* *}(F \circ G)=\mu^{* *}\left(F \circ{ }^{\prime} G\right) \\
& =\mu^{* *}(F) \circ \mu^{\prime *}(G)=F^{\prime} \circ{ }^{\prime} G^{\prime} .
\end{aligned}
$$

(b) Let $T \in M_{\varphi}(A)$ and $\left\{e_{\alpha}: \alpha \in I\right\}$ be a bounded approximate identity in $A$ (see [9]). A simple computation shows that $T=\lim _{\alpha T\left(e_{\alpha}\right)} \varphi$, which means $\overline{\mu(A)}=M_{\varphi}(A)$. So by Lemma 5 we conclude that $M_{\varphi}(A)$ is amenable.

Theorem 7. Let $A$ be a unital Banach algebra and let $\varphi: A \rightarrow$ $A$ be a spectrum preserving homomorphism with dense range. Then each $T \in M_{\varphi}(A)$ is spectrum preserving.

Proof. Let $a \in A$ and $\lambda \notin \sigma(T(a))$. Since $\varphi$ has dense range, there exists a sequence $\left\{c_{n}\right\}_{n} \in A$ such that $(T(a)-$ $\lambda) \lim _{n} \varphi\left(c_{n}\right)=1$. Thus

$$
\begin{aligned}
& (\varphi(a)-\lambda) \lim _{n} T\left(c_{n}\right) \\
& \quad=\lim _{n}(\varphi(a)-\lambda) T\left(c_{n}\right)=\lim _{n} \varphi(a-\lambda) T\left(c_{n}\right) \\
& \quad=\lim _{n} T(a-\lambda) \varphi\left(c_{n}\right)=(T(a)-\lambda) \lim _{n} \varphi\left(c_{n}\right) \\
& \quad=1 .
\end{aligned}
$$

Similarly $\lim _{n} T\left(c_{n}\right)(\varphi(a)-\lambda)=1$. Thus $\lambda \notin \sigma(\varphi(a))$. Since $\sigma(\varphi(a))=\sigma(a)$, we have $\lambda \notin \sigma(a)$.
Now, let $\lambda \notin \sigma(a)$. Then there exists $b \in A$ such that ( $a-$ $\lambda) b=1$. Thus

$$
(T(a)-\lambda) \varphi(b)=T(a-\lambda) \varphi(b)=T((a-\lambda) b)=1 .
$$

Similarly $\varphi(b)(T(a)-\lambda)=1$. Hence $\lambda \notin \sigma(T(a))$, which means $\sigma(T(a))=\sigma(a)$.

\section{3. $\varphi$-Multipliers on Topological Modules and Their Properties}

Now, we consider $\varphi$-multipliers in the general topological module setting and investigate some of their properties.

Definition 8. Let $A$ be a topological algebra and let $X, Y$ be two topological $A$-bimodules and let $\varphi$ be a nonzero and continuous idempotent $A$-module homomorphism on $X$. A linear and bounded mapping $T: X \rightarrow Y$ is called a left (resp., right) $\varphi$-multiplier if $T(a \cdot x)=T(\varphi(x)) \cdot a$ (resp., $T(x \cdot a)=$ $a \cdot T(\varphi(x)))$ for all $a \in A, x \in X$. We say $T$ is a $\varphi$-multiplier if it is both a left $\varphi$-multiplier and a right $\varphi$-multiplier.

We denote by $M_{\varphi}(X, Y)$ (resp., $\left.M_{\varphi}^{l}(X, Y), M_{\varphi}^{r}(X, Y)\right)$ the collection of all $\varphi$-multipliers (resp., left $\varphi$-multipliers, right $\varphi$-multipliers).

It is easy to check that $\varphi \in M_{\varphi}(X, X)$. So $M_{\varphi}(X, X) \neq\{0\}$.

Example 9. Let $A$ be a topological algebra, $X$ an $A$-bimodule, and $\varphi$ an idempotent $A$-module homomorphism on $X$. For each $a \in A$ the mapping ${ }_{a} \varphi: X \rightarrow X$ defined by ${ }_{a} \varphi(x)=$ $a \cdot \varphi(x)$ is a left $\varphi$-multiplier on $X$.

Proof. Let $a, b \in A$ and $x \in X$,

$$
\begin{aligned}
{ }_{a} \varphi(b \cdot x) & =a \cdot \varphi(b \cdot x)=a \cdot b \cdot \varphi(x)=b \cdot a \cdot \varphi(\varphi(x)) \\
& =b \cdot{ }_{a} \varphi(\varphi(x)), \\
{ }_{a} \varphi(x \cdot b) & =a \cdot \varphi(x \cdot b)=a \cdot \varphi(x) \cdot b=a \cdot \varphi(\varphi(x)) \cdot b \\
& ={ }_{a} \varphi(\varphi(x)) \cdot b .
\end{aligned}
$$

Hence ${ }_{a} \varphi \in M_{\varphi}(X, X)$.

In the sequel, $A$ denotes a topological algebra and $X, Y$ are two topological $A$-bimodules. In general, $\varphi$ is an $A$-module homomorphism on $X$ such that it is also idempotent, linear, and continuous. Sometimes $\varphi$ is on $A$; it will be mentioned when this happens.

Lemma 10. $M_{\varphi}^{l}(X, Y)$ is a left A-module.

Proof. $M_{\varphi}^{l}(X, Y)$ denotes the vector space of all left $\varphi$-multipliers from $X$ to $Y$. Let $T \in M_{\varphi}^{l}(X, Y)$ and $a \in A$ be arbitrary. Define $a * T$ as $(a * T)(x):=T(x \cdot a)$ where $x \in X$ is arbitrary. Since the equalities

$$
\begin{aligned}
(a * T)(b \cdot x) & =T(b \cdot x \cdot a)=b \cdot T(\varphi(x \cdot a)) \\
& =b \cdot T(\varphi(x) \cdot a)=b \cdot(a * T)(\varphi(x))
\end{aligned}
$$


hold for all $b \in A$ and $x \in X$, we conclude that $a * T$ is a left $\varphi$-multiplier. Then, since $X$ is an $A$-bimodule and $T$ is linear, $M_{\varphi}^{l}(X, Y)$ is a left $A$-module.

Definition 11. An $A$-bimodule $X$ is said to be commutative if $a \cdot x=x \cdot a$ holds for all $a \in A$ and $x \in X$.

Definition 12 (see $[10,11])$. Let $X$ be a left (resp., right) $A$ module. $A$ is said to be left (resp., right) faithful in $X$ if, for any $x \in A, a \cdot x=0$ (resp., $x \cdot a=0$ ) for all $a \in A$ implies that $x=0$. If $X$ is an $A$-bimodule then $A$ is said to be faithful in $X$ if it is both left and right faithful in $X$.

The following definition generalizes Definition 12.

Definition 13. Let $X$ be a left (resp., right) $A$-module. $A$ is said to be left (resp., right) $\varphi$-faithful in $X$ if, for any $x \in A, \varphi(a)$. $x=0$ (resp., $x \cdot \varphi(a)=0$ ) for all $a \in A$ implies that $x=0$. If $X$ is an $A$-bimodule then $A$ is said to be $\varphi$-faithful in $X$ if it is both left and right $\varphi$-faithful in $X$.

Definition 14. Let $A$ be a topological algebra, $X$ a commutative $A$-bimodule, and $\varphi$ an idempotent $A$-module homomorphism on $A$. For any $x \in X$, define

$$
{ }_{x} \varphi: A \longrightarrow X \text { by }{ }_{x} \varphi(a)=x \cdot \varphi(a), a \in A .
$$

It is easy to see that ${ }_{x} \varphi \in M_{\varphi}^{l}(A, X)$. Now, we define $\psi: X \rightarrow$ $M_{\varphi}^{l}(A, X)$, by $\psi(x)={ }_{x} \varphi$.

Lemma 15. Let $A$ be a topological algebra with an approximate identity $\left\{e_{\lambda}: \lambda \in I\right\}$ and let $X$ be a topological $A$ bimodule. If $A$ is $\varphi$-faithful in $X$ and $\psi: X \rightarrow M_{\varphi}^{l}(A, X)$ is onto, then $A$ is left faithful in $M_{\varphi}^{l}(A, X)$.

Proof. Let $T \in M_{\varphi}^{l}(A, X)$. In view of Lemma 10, it is enough to show that $T=0$ if $a * T=0$ for all $a \in A$. Since $\psi$ is onto, there exist $x \in X$ such that $T={ }_{x} \varphi$. Therefore for any $b \in A$ and $\lambda \in I$,

$$
x \cdot \varphi\left(b \cdot e_{\lambda}\right)={ }_{x} \varphi\left(b \cdot e_{\lambda}\right)=\left(e_{\lambda} *{ }_{x} \varphi\right)(b)=0 .
$$

The continuity of $\varphi$ implies that $x \cdot \varphi(b)=0$. Now, since $A$ is $\varphi$ faithful in $X$, we conclude that $x=0$. Hence $T={ }_{x} \varphi=0$.

Definition 16. Let $A$ be a Hausdorff topological algebra and $(X, \tau)$ a Hausdorff topological $A$-bimodule. Let $\varphi$ be an $A$-module homomorphism on $A$. The $\varphi$-uniform operator topology $u_{\varphi}$ (resp., $\varphi$-strong operator topology $\left.s_{\varphi}\right)$ on $M_{\varphi}^{l}(A$, $X)$ is defined as the linear topology which has a base of neighborhoods of 0 consisting of all sets of the form

$$
N(\varphi(B), V)=\left\{T \in M_{\varphi}^{l}(A, X): T(\varphi(B)) \subseteq V\right\},
$$

where $B$ is a bounded (resp., finite) subset of $A$ and $V$ is a neighborhood of 0 in $X$. Clearly $s_{\varphi} \leq u_{\varphi}$.

Theorem 17. Let $\varphi$ be an A-module homomorphism on $A$ and let $(X, \tau)$ be a topological A-bimodule with $b(A)$-hypocontinuous module multiplication.
Then $\left(M_{\varphi}^{l}(A, X), s_{\varphi}\right)$ and $\left(M_{\varphi}^{l}(A, X), u_{\varphi}\right)$ are topological left A-modules.

Proof. By Lemma 10, $M_{\varphi}^{l}(A, X)$ is a left $A$-module. Now, let us prove that the module multiplication $(a, T) \rightarrow a * T$ from $A \times M_{\varphi}^{l}(A, X)$ into $M_{\varphi}^{l}(A, X)$ is separately continuous in $u_{\varphi^{-}}$ topology. Let $T \in M_{\varphi}^{l}(A, X)$ and $\left\{a_{\alpha}: \alpha \in I\right\}$ be a net in $A$ with $a_{\alpha} \rightarrow a \in A$ and let $D$ be a bounded subset of $A$ and let $V$ be a neighborhood of 0 in $X$. By $b(A)$-hypocontinuity, there exists a balanced neighborhood $H$ of 0 in $X$ such that $\varphi(D) \cdot H \subset V$. Since $T$ and $\varphi$ are continuous, there exist $\alpha_{0} \in I$ such that

$$
\begin{aligned}
& \left(a_{\alpha} * T\right)(\varphi(b))-(a * T)(\varphi(b)) \\
& \quad=T\left(\varphi(b) \cdot a_{\alpha}\right)-T(\varphi(b) \cdot a) \\
& =\varphi(b) \cdot\left[T\left(\varphi\left(a_{\alpha}\right)\right)-T(\varphi(a))\right] \\
& \quad \in \varphi(D) \cdot H \subset V
\end{aligned}
$$

for all $b \in D$ and $\alpha \geq \alpha_{0}$. Hence $a_{\alpha} * T \rightarrow{ }_{u_{\varphi}} a * T$.

Next, let $a \in A$ and $\left\{T_{\alpha}: \alpha \in I\right\}$ be a net in $M_{\varphi}^{l}(A, X)$ such that $T_{\alpha} \rightarrow{ }_{u_{\varphi}} T \in M_{\varphi}^{l}(A, X)$ and let $D$ be a bounded subset of $A$ and let $V$ be a neighborhood of 0 in $X$. Since the mappings $\varphi$ and $R_{a}(x)=x a$ are continuous, it follows that $\varphi(D) \cdot a$ is a bounded subset in $A$. So there exist $\alpha_{0} \in I$ such that

$$
\begin{aligned}
\left(a * T_{\alpha}-a * T\right)(\varphi(b)) & =T_{\alpha}(\varphi(b) \cdot a)-T(\varphi(b) \cdot a) \\
& =\left(T_{\alpha}-T\right)(\varphi(D) \cdot a) \subseteq V
\end{aligned}
$$

for all $\alpha \geq \alpha_{0}$ and $b \in D$. Hence $a * T_{\alpha} \rightarrow{ }_{u_{\varphi}} a * T$. That means $\left(M_{\varphi}^{l}(A, X), u_{\varphi}\right)$ is a left topological module. A similar computation shows that $\left(M_{\varphi}^{l}(A, X), s_{\varphi}\right)$ is a left topological module.

Lemma 18. Let $A$ be a topological algebra with an approximate identity $\left\{e_{\lambda}: \lambda \in I\right\}$ and let $(X, \tau)$ be a commutative A-bimodule and $\varphi$ an idempotent A-module homomorphism on $A$. Then $\overline{\psi(X)^{s_{\varphi}}}=M_{\varphi}^{l}(A, X)$.

Proof. Let $T \in M_{\varphi}^{l}(A, X)$, and let $B$ be a finite subset of $A$ and let $V$ be a neighborhood of 0 in $X$. For each $a \in A$ we have $e_{\lambda} \varphi(a) \rightarrow \varphi(a)$. Then, since $T$ is continuous and $B$ is finite, there exist $\lambda_{0} \in I$ such that $T\left(e_{\lambda} \varphi(a)\right)-T(\varphi(a)) \in V$, for all $a \in B$ and $\lambda \geq \lambda_{0}$. Then, for any $a \in B$ and $\lambda \geq \lambda_{0}$

$$
\begin{aligned}
& \left\{T\left(\varphi\left(e_{\lambda}\right)\right)\right\}_{\lambda} \varphi(\varphi(a))-T(\varphi(a)) \\
& \quad=T\left(\varphi\left(e_{\lambda}\right)\right) \cdot \varphi(\varphi(a))-T(\varphi(a)) \\
& =T\left(e_{\lambda} \varphi(a)\right)-T(\varphi(a)) \in V .
\end{aligned}
$$

Therefore $_{\left\{T\left(\varphi\left(e_{\lambda}\right)\right)\right\}_{\lambda}} \varphi \rightarrow{ }_{s_{\varphi}} T$.

Theorem 19. Let $A$ be a topological algebra with an approximate identity $\left\{e_{\lambda}: \lambda \in I\right\}$ and let $X$ be a commutative 
topological A-bimodule such that $(X, \tau)$ is complete. Suppose $\varphi$ is an idempotent $A$-module homomorphism on $A$ and $A$ is $\varphi$-faithful in $X$. Then $(X, \tau)$ is isomorphic to $\left(M_{\varphi}^{l}(A, X), s_{\varphi}\right)$.

Proof. Let $\psi$ be as in Definition 14. It is obvious that $\psi$ is a continuous module homomorphism. We first show that $\psi$ is onto. In view of Lemma 18 , it is enough to prove that $\psi(X)$ is $s_{\varphi}$-closed. Let $T \in \overline{\psi(X)}^{s_{\varphi}}$. Then there exists a net $\left\{x_{\alpha}\right\}_{\alpha} \subseteq X$ such that ${ }_{x_{\alpha}} \varphi \rightarrow{ }_{s_{\varphi}} T$. It follows that the net $\left\{x_{\alpha} \cdot \varphi(a)\right\}_{\alpha}=$ $\left\{_{x_{\alpha}} \varphi(\varphi(a))\right\}_{\alpha}$ is $\tau$-Cauchy in $X$ for every $a \in A$. Now, since $A$ is $\varphi$-faithful in $X$, the net $\left\{x_{\alpha}\right\}$ is $\tau$-Cauchy in $X$. By completeness of $(X, \tau)$, there exist $x \in X$ such that $x_{\alpha} \rightarrow x$. Hence ${ }_{x_{\alpha}} \varphi \rightarrow{ }_{s_{\varphi} x} \varphi$. By uniqueness of limit in Hausdorff space $T=$ ${ }_{x} \varphi$. Therefore $\psi(X)$ is $s_{\varphi}$-closed.

To show that $\psi$ is one-to-one, let $x, y \in X$ such that ${ }_{x} \varphi=$ ${ }_{y} \varphi$. Then for any $a \in A,(x-y) \cdot \varphi(a)=0$. Since $A$ is $\varphi$-faithful in $X$, this implies that $x=y$. Thus $\psi$ is one-to-one.

Definition 20. Let $A$ be a topological algebra and let $X$ be a topological $A$-bimodule. The uniform topology $\gamma_{\varphi}$ (strict topology $\left.\beta_{\varphi}\right)$ on $M_{\varphi}^{l}(A, X)$ is defined as the linear topology which has a base of neighborhoods of 0 consisting of all sets

$$
N^{\prime}(\varphi(D), G)=\left\{T \in M_{\varphi}^{l}(A, X): \varphi(D) * T \subset G\right\},
$$

where $D$ is a bounded (finite) subset of $A$ and $G$ is a neighborhood of 0 in $\left(M_{\varphi}^{l}(A, X), u_{\varphi}\right)\left[\left(M_{\varphi}^{l}(A, X), s_{\varphi}\right)\right]$.

Lemma 21. Let $A$ be a topological algebra with a bounded approximate identity $\left\{e_{\lambda}: \lambda \in I\right\}$ and let $X$ be a topological A-bimodule. Then $u_{\varphi}=\gamma_{\varphi}$ and $s_{\varphi}=\beta_{\varphi}$.

Proof. Let $\left\{T_{\alpha}\right\}_{\alpha}$ be a net in $M_{\varphi}^{l}(A, X)$ with $T_{\alpha} \rightarrow{ }_{u_{\varphi}} T$. Let $G=$ $N(\varphi(C), V)$ be a neighborhood of 0 in $u_{\varphi}$-topology. Since $\varphi$ is continuous, $\varphi(C) \varphi(D)$ is a bounded subset of $A$ for each bounded subset $D$ of $A$. Then there exist $\alpha_{0}$ such that

$$
\left(\varphi(D) *\left(T_{\alpha}-T\right)\right)(\varphi(C))=\left(T_{\alpha}-T\right)(\varphi(C) \varphi(D)) \in V
$$

for all $\alpha \geq \alpha_{0}$. That means $\varphi(D) *\left(T_{\alpha}-T\right) \in G$. Hence $T_{\alpha} \rightarrow{ }_{\gamma_{\varphi}} T$.

Conversely, let $\left\{T_{\alpha}\right\}_{\alpha}$ be a net in $M_{\varphi}^{l}(A, X)$ with $T_{\alpha} \rightarrow{ }_{\gamma_{\varphi}} T$. Let $D$ be a bounded subset of $A$ and let $V$ be a closed neighborhood of 0 in $X$. Choose $C=\left\{e_{\lambda}\right\}_{\lambda}$. Then there exist $\alpha_{0}$ such that

$$
\begin{aligned}
\left(T_{\alpha}-T\right)(\varphi(D)) & =\lim _{\lambda}\left(T_{\alpha}-T\right)(\varphi(C) \varphi(D)) \\
& =\lim _{\lambda}\left(\varphi(D) *\left(T_{\alpha}-T\right)\right)(\varphi(C)) \in V
\end{aligned}
$$

for all $\alpha \geq \alpha_{0}$. That means $T_{\alpha}-T \in N(\varphi(D), V)$. Hence $T_{\alpha} \rightarrow{ }_{u_{\varphi}} T$.

At the end we characterize the $\varphi$-multipliers on $L_{p}(G)$, where $G$ is a compact Abelian group. Of course, $L_{p}(G)$ is a
Banach algebra and several authors studied its multipliers. For instance, Larsen [5] showed that a linear transformation $T: L_{p}(G) \rightarrow L_{p}(G)$, where $G$ is a locally compact Abelian group, is a multiplier if and only if there exists a unique $\varphi \in$ $L_{\infty}(\widehat{G})$ such that $\widehat{T f}=\varphi \widehat{f}$ for each $f \in L_{p}(G)$.

However, we now consider $L_{p}(G)$ as a left Banach module over the group algebra $L_{1}(G)$. Namely, the algebra $L_{1}(G)$ acts on $L_{p}(G)$ through the convolution $L_{1}(G) * L_{p}(G)=L_{p}(G)$.

Example 22. Let $G$ be a compact Abelian group and let $\varphi$ : $L_{1}(G) \rightarrow L_{p}(G)$ be an idempotent $L_{1}(G)$-module homomorphism with dense range. If $T: L_{p}(G) \rightarrow L_{p}(G)$ is a $\varphi$-multiplier then there exists a unique function $H_{T} \in L_{p}(G)$ such that

$$
T(\varphi(f))=\varphi(f) * H_{T}, \quad\left(f \in L_{1}(G)\right) .
$$

Proof. Let $\left\{e_{\beta}\right\}_{\beta}$ be a bounded approximate identity in $L_{1}(G)$. Then $\left\{T\left(\varphi\left(e_{\beta}\right)\right)\right\}_{\beta} \subseteq \operatorname{Ball}\left(L_{q}(G)\right)^{*}$. By the Alaoglu theorem, there exists a function $H_{T} \in L_{p}(G)$ such that $T\left(\varphi\left(e_{\beta}\right)\right) \rightarrow{ }_{\text {weak* }} H_{T}$. Then for each $f \in L_{1}(G)$

$$
\varphi(f) * T\left(\varphi\left(e_{\beta}\right)\right) \longrightarrow_{\text {weak } *} \varphi(f) * H_{T} .
$$

On the other hand, since $T$ is a $\varphi$-multiplier,

$$
\varphi(f) * T\left(\varphi\left(e_{\beta}\right)\right)=T\left(\varphi\left(f * e_{\beta}\right)\right) \longrightarrow T(\varphi(f))
$$

for each $f \in L_{1}(G)$. By uniqueness of limit, $T(\varphi(f))=\varphi(f) *$ $H_{T}$.

To show that $H_{T}$ is unique, let $\psi$ be a second function in $L_{p}(G)$ such that $T(\varphi(f))=\varphi(f) * \psi$ for each $f \in L_{1}(G)$. Since $\varphi$ has dense range, $T(\varphi(f))=f * \psi$ for each $f \in L_{1}(G)$. Therefore

$$
\widehat{f}(\gamma)\left({\widehat{\psi-H_{T}}}\right)(\gamma)=0
$$

for each $f \in L_{1}(G)$ and $\gamma \in \widehat{G}$. By compactness of $G$, for each $\gamma \in \widehat{G}$ there exist $f \in L_{1}(G)$ such that $\widehat{f}(\gamma) \neq 0$. Hence the semisimplicity of $L_{p}(G)$ implies that $H_{T}=\psi$.

\section{Conflict of Interests}

The author declares that there is no conflict of interests regarding the publication of this paper.

\section{Acknowledgment}

The author wishes to thank the referee for several useful suggestions which significantly improved the quality of the paper.

\section{References}

[1] S. Helgason, "Multipliers of Banach algebras," Annals of Mathematics, vol. 64, pp. 240-254, 1956.

[2] J.-K. Wang, "Multipliers of commutative Banach algebras," Pacific Journal of Mathematics, vol. 11, pp. 1131-1149, 1961. 
[3] F. T. Birtel, "Isomorphisms and isometric multipliers," Proceedings of the American Mathematical Society, vol. 13, pp. 204-210, 1962.

[4] A. Riazi and M. Adib, " $\varphi$-multipliers on Banach algebras without order," International Journal of Mathematical Analysis, vol. 3, no. 1-4, pp. 121-132, 2009.

[5] R. Larsen, An Introduction to The Theory of Multipliers, Springer, New York, NY, USA, 1970.

[6] B. Zalar, "On centralizers of semiprime rings," Commentationes Mathematicae Universitatis Carolinae, vol. 32, no. 4, pp. 609614, 1991.

[7] J. Vukman, "An identity related to centralizers in semiprime rings," Commentationes Mathematicae Universitatis Carolinae, vol. 40, pp. 255-265, 2001.

[8] F. F. Bonsall and J. Duncan, Complete Normed Algebras, Springer, Berlin, Germany, 1973.

[9] V. Runde, Lectures on Amenability, vol. 1774 of Lecture Notes in Mathematics, Springer, Berlin, Germany, 2002.

[10] B. E. Johnson, "An introduction to the theory of centralizers," Proceedings of the London Mathematical Society, vol. 14, pp. 299320, 1964.

[11] F. D. Sentilles and D. C. Taylor, "Factorization in Banach algebras and the general strict topology," Transactions of the American Mathematical Society, vol. 142, pp. 141-152, 1969. 


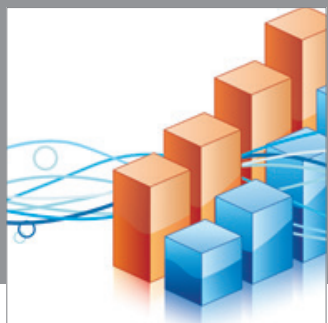

Advances in

Operations Research

mansans

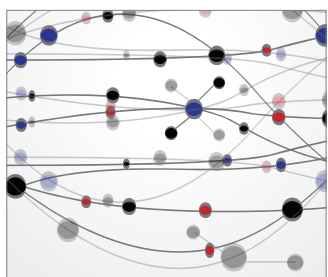

The Scientific World Journal
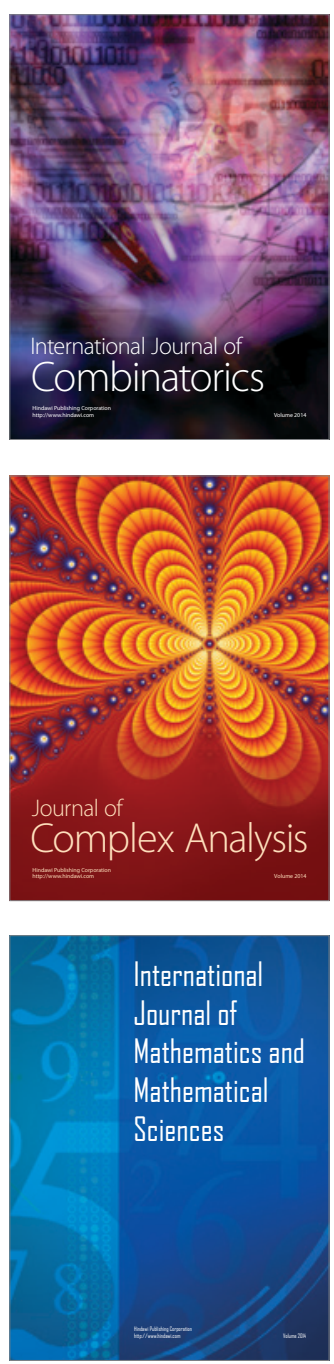
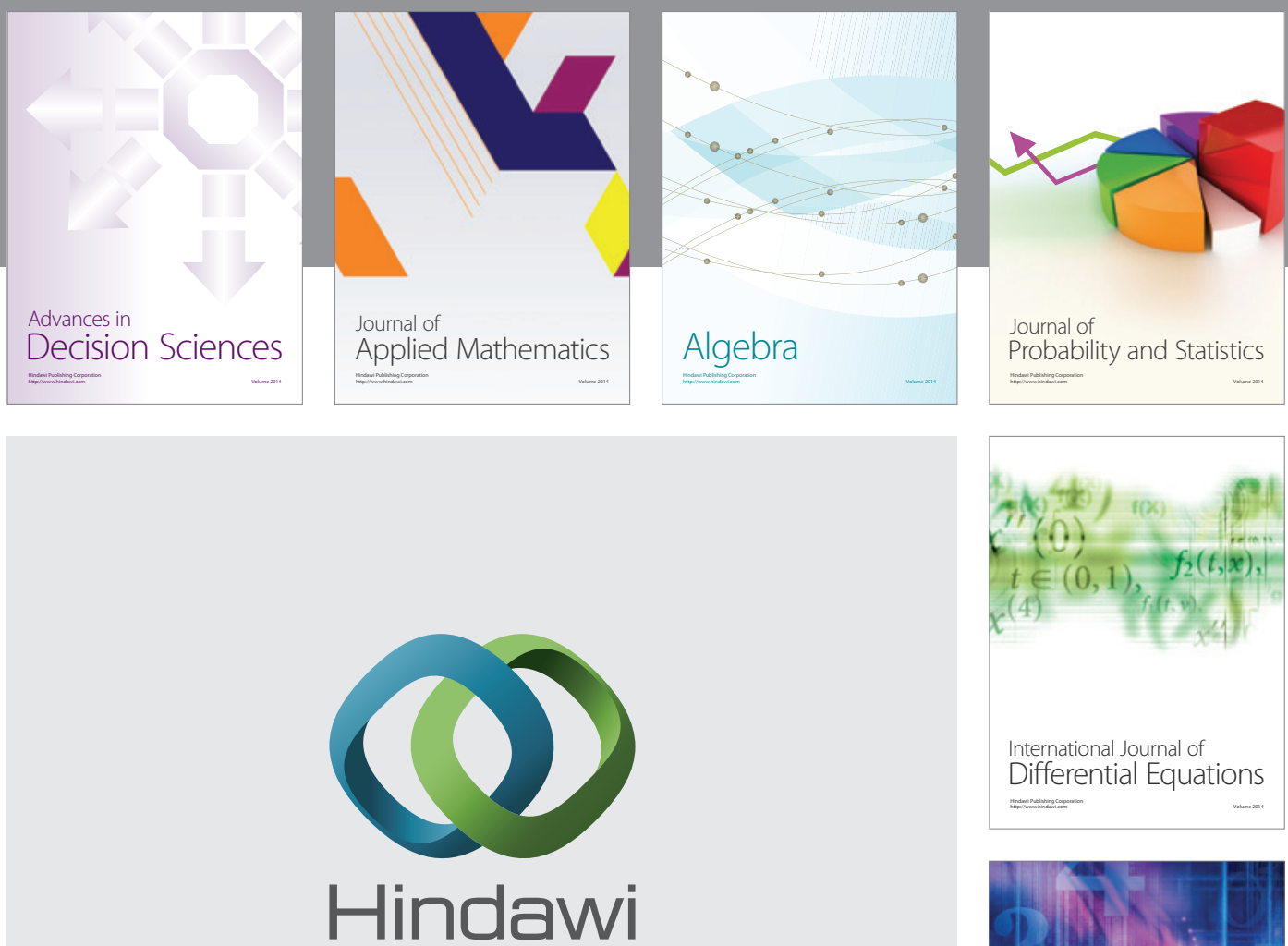

Submit your manuscripts at http://www.hindawi.com
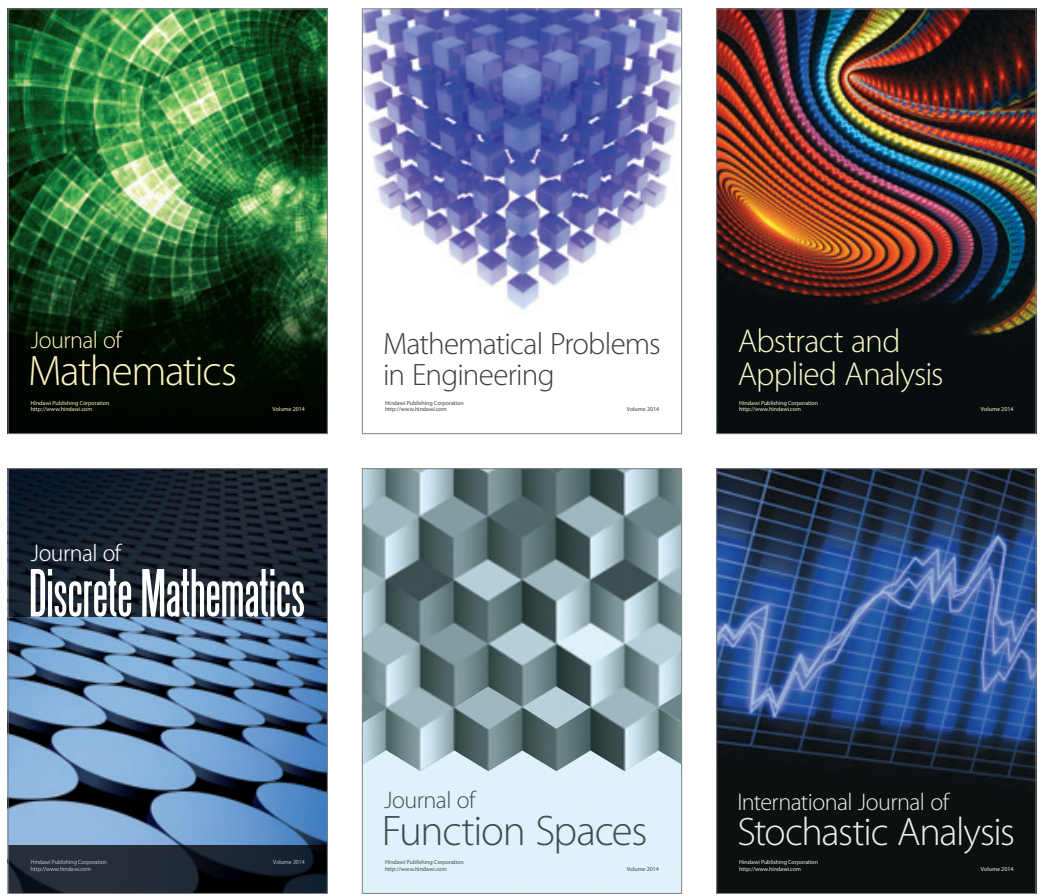

Journal of

Function Spaces

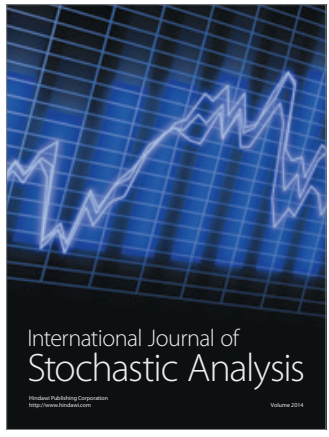

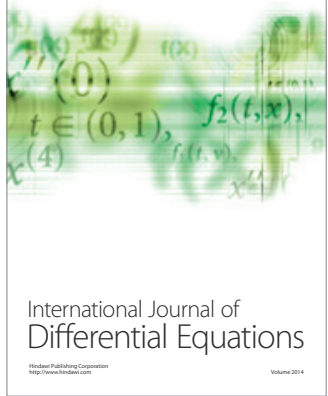
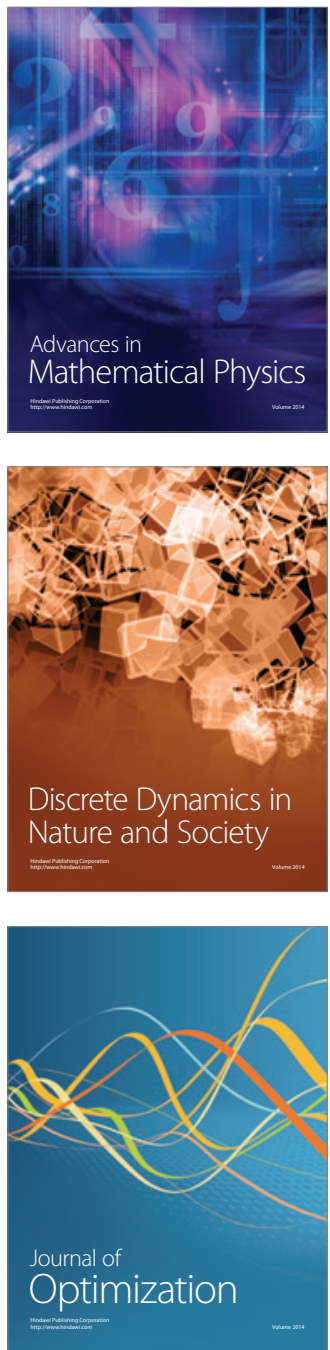